\title{
Parametric Study on Dynamic Tension Behaviour of Offshore Pipeline for Deepwater S-lay Operation
}

\author{
$\mathrm{Pu} \mathrm{XU}^{1}$, Shun Feng $\mathrm{GONG}^{2}$, Ting $\mathrm{ZHANG}^{1}$, Cheng $\mathrm{CHEN}^{1}$ \\ ${ }^{1}$ College of Civil Engineering, Fuzhou University, Fuzhou 350116, China \\ ${ }^{2}$ Institute of Structural Engineering, Zhejiang University, Hangzhou 310058, China
}

\begin{abstract}
In recent years, higher demands for offshore pipeline design of deepwater installation are put forward. This paper mainly aims to investigate the influence of pipelay parameters on dynamic tension behaviour of deepwater S-lay pipeline to optimize the design of pipe structure and tensioner. A full finite element model for deepwater S-lay systems is developed to simulate the dynamic response of offshore pipeline from the pipelay vessel via stinger to the seabed. The influences of water depth, outer diameter and submerged self-weight of the pipe, and stinger length on the dynamic tension behaviour of the pipeline have been quantitatively studied. A strong relevance between the axial tension of offshore pipeline and pipelay parameters is observed, which offers very intuitive evidences for the design of pipe structure and tensioners.
\end{abstract}

\section{Introduction}

With the exploration of offshore oil-gas resources expanding into deep waters, pipelines play an indispensable role of large-scale transportation for crude oil and natural gas. S-lay technique is widely applied to install the pipeline from sea level to the seafloor due to its advantages of higher pipelay efficiency and better workability [1]. In the S-lay method, the pipeline passes through the tensioners and slides over the stinger at the stern of the vessel via a sloping ramp before reaching the seabed, as shown in Figure 1. The tensioners mounted on the lay vessel are functioned to apply a horizontal tension to the pipe at the top end and control the pipeline configuration in the sagbend. The axial tension response of the pipeline in the deepwater S-lay process is very significant and variable, and directly dominates the design of the pipe structure and tensioners. Analytical and numerical solutions were developed to carry out the dynamic response analysis of offshore pipeline for S-lay operation [2-4]. Mattiazzo et al. [5] proposed a numerical model to analyze the performance of the tensioner used in the process of pipeline installation. Yue et al. [6] presented an analytical model to predict the tension behavior of the flexible pipeline in shallow water. Lately, Gong et al. [78] developed a full element model to simulate the dynamic response of deepwater S-lay pipeline. The objective of the present study is to explore the influence of pipelay parameters on the axial tension behavior of offshore pipeline for deepwater S-lay operation, and promote the optimal design of pipe structure and tensioners.

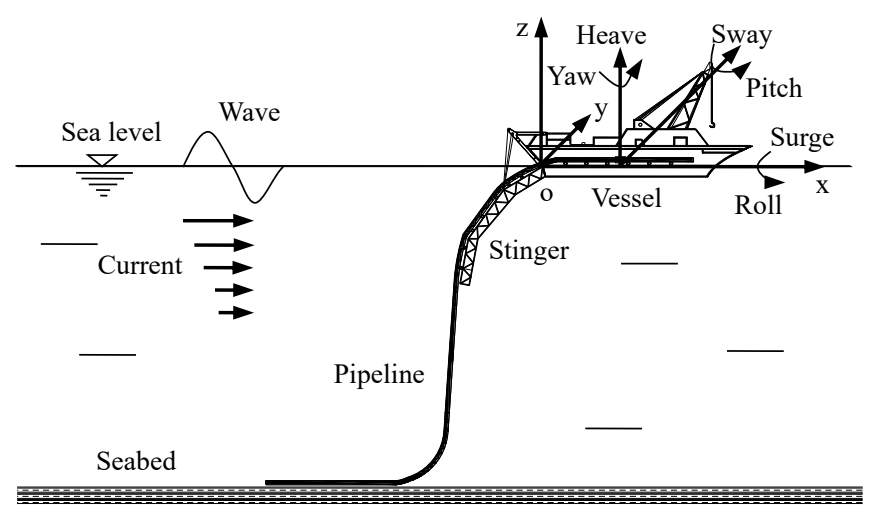

Figure 1 Deepwater S-lay pipeline

\section{Theory Models}

\footnotetext{
*Corresponding author: Email: puxu@fzu.edu.cn
} 
A comprehensive finite model for deepwater S-lay system is established to simulate the tension behavior of offshore pipeline based upon the commercial commercial computer code OrcaFlex [9]. The effectiveness and practicality of the model is adequately validated through a practical engineering example of a 6 -inch pipeline laying to the LW3-1 gas filed in the South China Sea [7]. In this model,

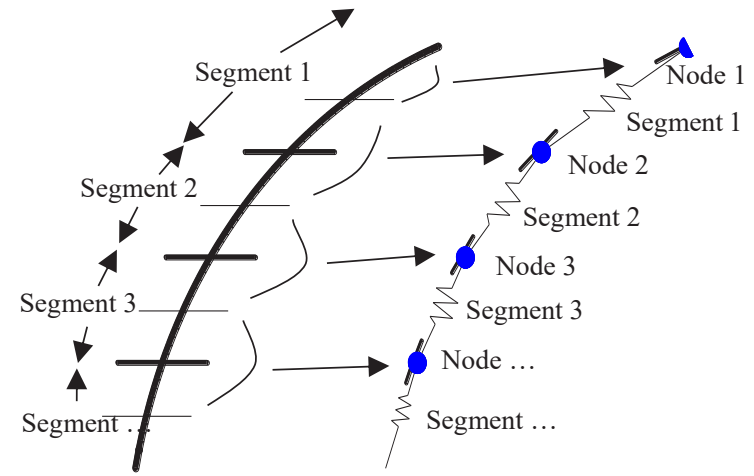

Figure 2 Finite element model of pipeline structure [9]

An axial spring and a damper at the center of line segment is used to simulate the axial stiffness and damping of the pipeline, and applies an equal and opposite effective tension force to the nodes at each end of line segment. Meanwhile, it is assumed that the line segment is subjected to external and internal fluid pressure, as shown in Figure 3, the effective tension at the mid-point of line segment can be expressed as

$$
T_{\mathrm{e}}=T_{\mathrm{w}}(\varepsilon)+(1-2 v) \cdot\left(P_{\mathrm{o}} A_{\mathrm{o}}-P_{\mathrm{i}} A_{\mathrm{i}}\right)+E A_{\text {nom }} \cdot \xi \cdot(d L / d t) / L_{0}
$$

the pipeline is divided into a series of line segments which are modelled by straight massless model segments with a node at each end. A representative single mid-line node and line segments either side of it are selected to establish the local xyz-frame frames of reference, as illustrated in Figure 2.

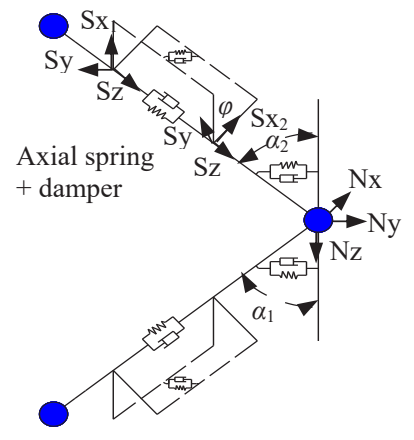

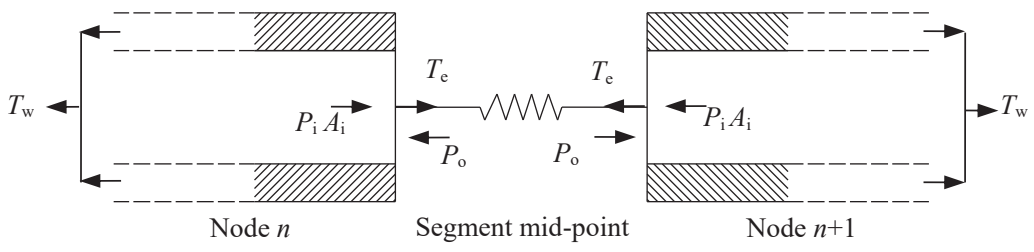

Figure 3 Tension analysis of the pipe at the mid-point of line segment [9]

The non-linear stress-strain relationship of the steel pipe is characterized by Ramberg-Osgood model [10], which is expressed as

$$
\varepsilon(\sigma)=\sigma / E+B\left(\sigma / \sigma_{\mathrm{y}}\right)^{n}
$$

Where $B$ and $n$ are the coefficient and power exponent constants that depend on the steel material grade.

\subsection{Interaction Models}

At the top end, the pipeline is usually supported by the tensioner and multiple discrete rollers on the main work line of the pipelay vessel, as shown in Figure 4. The
Where $T_{\mathrm{w}}$ is the function relating axial strain to wall tension, $P_{\mathrm{o}}$ and $P_{\mathrm{i}}$ are external and internal pressure, $A_{\mathrm{o}}$ and $A_{\mathrm{i}}$ are external and internal cross-section areas of the stress annulus, $v$ is the Poisson's ratio, $E A_{\text {nom }}$ is the nominal axial stiffness which is defined to be the axial stiffness at zero strain, $L$ and $L_{\mathrm{o}}$ are the instantaneous and unstretched length of line segment, $\xi$ is the axial damping coefficient. tension winch element attached to the vessel and the pipe is adopted to simulate the tensioner, ensuring that the effective tension fed from the tensioner is applied to the top end of the pipeline. The parts of the pipeline in the overbend are supported by the roller boxes on the stinger hinged off the back of the HYSY 201 pipelay vessel. The S-lay stinger is made up of three sections of truss structures mutually connected via hinges, and on which 10 roller boxes are regularly-spaced and mounted. Each roller in the roller box should be examined to identify whether the pipeline is in contact with the roller. If the pipeline is confirmed to be touching the roller, the support reaction is then calculated and applied to the pipe and the roller.
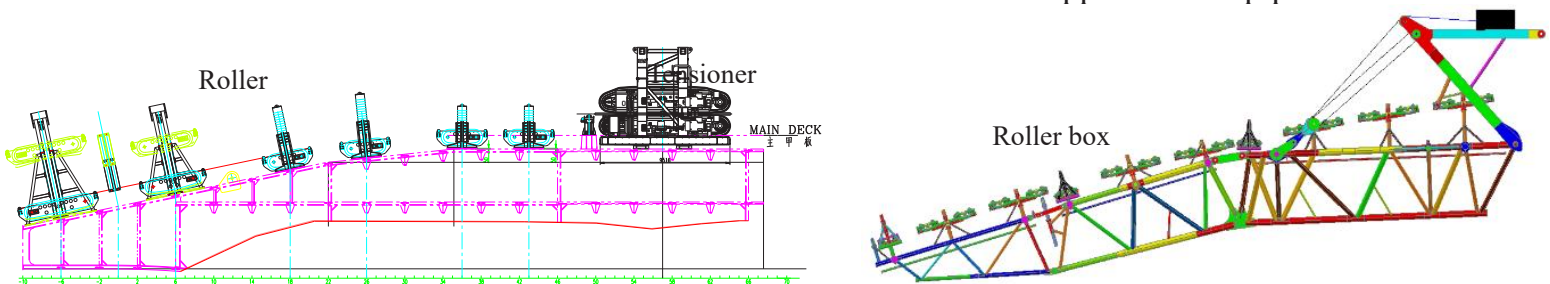

Figure 4 Main work line (a) and articulated stinger (b) at the stern of the HYSY201 pipelay vessel

At the bottom, the pipeline is gradually laid on the seabed during deepwater installation. The pipe-seabed 
vertical interaction under cyclic motions is modelled by a non-linear seabed model based on a hyperbolic secant stiffness formulation [11], as shown in Figure 5. In this model, four penetration modes, namely not-in-contact, initial penetration, uplift and repenetration, are used to represent the variation of the pipeline motion on the seabed. In each penetration mode, the seabed normal resistance per unit length, $P(z)$, is modelled by an analytic function of the non-dimensionalized penetration, $z / D$, where $z$ is the penetration and $D$ is the pipe diameter. On the other side, the pipe-seabed lateral interaction is simulated by the modified Coulomb friction model, as illustrated in Figure 6. Based upon the seabed soil type, the lateral seabed resistance in the model is represented as the product of the vertical seabed reaction force per unit length $P(z)$ and a soil friction coefficient $u$. The friction force is assumed to be linear variation from $-u P(z)$ to $+u P(z)$ as the deflection ranges from $-\mathrm{y}$ breakout to $+\mathrm{y}$ breakout, and given by $F_{\mathrm{y}}=-k_{\mathrm{s}} A y$ to a magnitude of not more than $u P(z)$, where $y$ is the displacement from the un-sheared position, $k_{\mathrm{s}}$ is the seabed shear stiffness, and $A$ is the contact area.

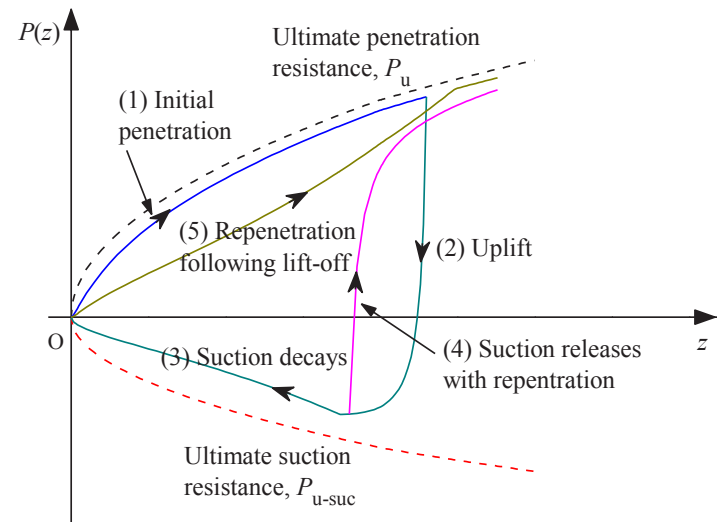

Figure 5 Non-linear hysteretic soil model [11]

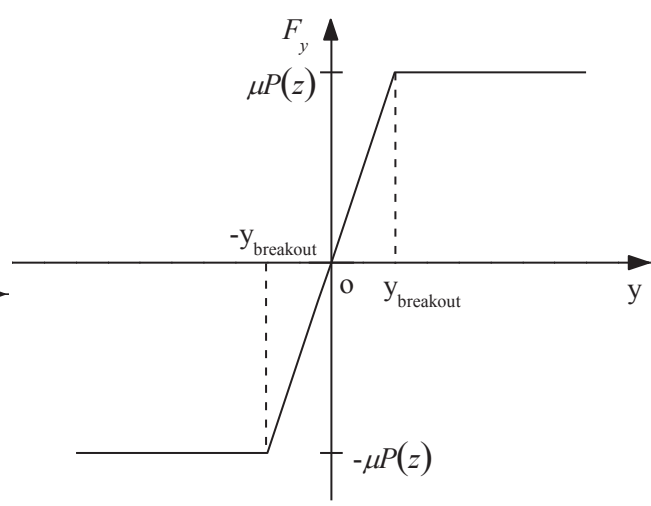

Figure 6 Coulomb friction model

\subsection{Vessel Motions}

The vessel model for deepwater S-lay pipeline is based upon the HYSY 201 pipelay vessel with $204.65 \mathrm{~m}$ in length and $39.2 \mathrm{~m}$ in breadth. The vessel generally has 6 degrees of freedom at the center of gravity, i.e. 3 translations (surge, sway, heave) and 3 rotations (roll, pitch, yaw), as illustrated in Figure 1. The vessel motions induced by surface waves can be calculated by the displacement response amplitude operators (RAOs). Each displacement RAO is composed of a pair of numbers that define the vessel response to one particular degree of freedom for one particular wave direction and period.

\section{Numerical Implementation}

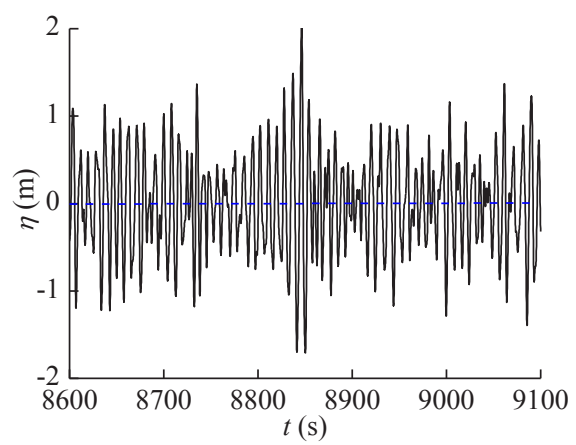

Figure 7 Wave time history for $H \mathrm{~s}=2.0 \mathrm{~m}$

\subsection{Metocean Conditions}

Based upon the marine environmental statistics in the gas field of the South China Sea, the standard wave spectrum JONSWAP is selected to represent the sea state with significant wave height $H_{\mathrm{s}}=2.0 \mathrm{~m}$ and peak period $T_{\mathrm{p}}=8.7$ $\mathrm{s}$. The equal energy method is adopted to discretize the wave spectrum, and one hundred linear wave components are then generated to construct an irregular random wave train by linear superposition, as illustrated in Figure 7. In order to cove the time when the maximum wave height $3.76 \mathrm{~m}$ occurs, the simulation time origin of the pipelay system is shifted to $8600 \mathrm{~s}$ with the specified simulation duration $500 \mathrm{~s}$. The vertical profile curve of current velocity based on the practical measurement in the gas field is illustrated in Figure 8 with surface current speed $1.0 \mathrm{~m} / \mathrm{s}$. The current direction is assumed to be coincident with the lay vessel heading.

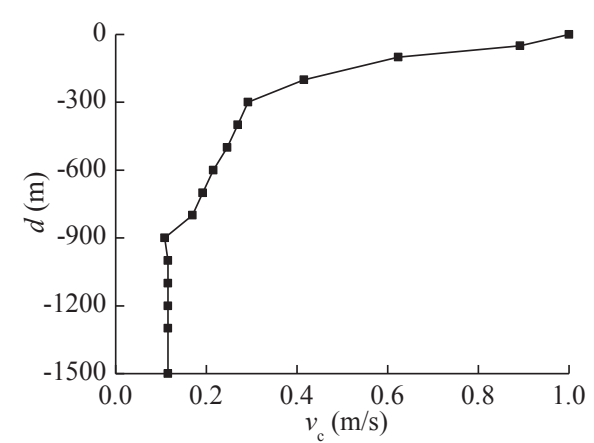

Figure 8 Current velocity distribution 


\subsection{Calculation Methods}

The whole calculation analysis for dynamic response of deepwater S-lay pipelines contains two steps, the first step is to conduct static analysis based upon catenary solution and determine the equilibrium position of pipelay system under weight, buoyancy, hydrodynamic drag, etc, the full static calculation is subsequently implemented particularly considering the effects of bending stiffness and interaction with solid shapes. The second step is to carry out dynamic analysis starting from the static equilibrium position. The explicit integration scheme with a constant time step is adopted to simulate the dynamic response of offshore pipeline, which sufficiently considers various geometric non-linearities and the spatial variation of both wave loads and contact loads. The axial numerical damping in a pipe segment consists of critical damping and target damping, which is given by $\xi=\xi_{\mathrm{c}} \cdot \xi_{\mathrm{t}}$. The axial critical damping for a line segment is expressed as $\xi_{\mathrm{c}}=\left(2 \cdot m_{\mathrm{s}} \cdot L_{0} / E A_{\text {nom }}\right)^{1 / 2}$, and the axial target damping is specified the value of $10 \%$. The numerical damping is capable of effectively damping down the high frequency noise and has litter influence on the calculation results of

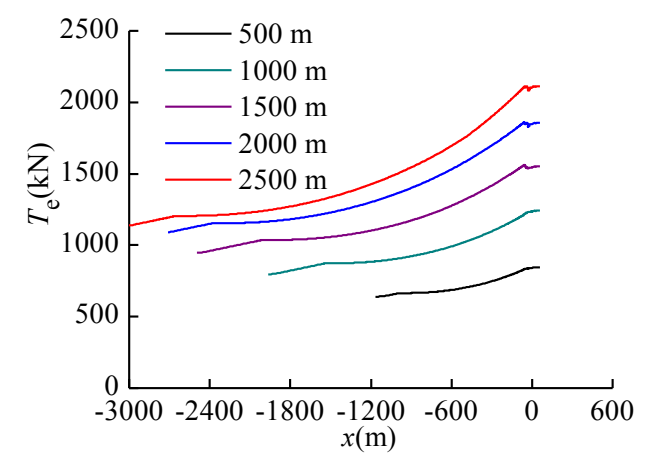

(a) Axial tension distribution along the pipeline

Figure 9 Axial tension response of the pipeline varied with water depth the pipelay systems.

\section{Result and Discussion}

\subsection{Tension Varied with Water Depth}

According to the practical engineering case in the LW3-1 gas field in the South China Sea, the pipeline need to laid with diameter $168.3 \mathrm{~mm}$, wall thickness $15.9 \mathrm{~mm}$, and a submerged weight $361.0 \mathrm{~N} / \mathrm{m}$. The response analyses of offshore pipeline in the process of S-lay operation are carried out under water depths being 500, 1000, 1500, 2000, and $2500 \mathrm{~m}$, respectively. As shown in Figure 9, with the increase of water depth, the axial tension of the entire pipeline significantly increases. A dynamic amplification factor (DAF) is defined as the dynamic response of the pipeline relative to the static response. The maximum axial tensions of the pipeline for both static and dynamic analysis linearly increases, whereas the corresponding DAF gradually drops. It is evidently illustrated from these results that the water depth has a great influence on axial tension of the pipeline, and the increase of water depth weakens the dynamic lay effect on maximum axial tension of the pipeline to some extent.

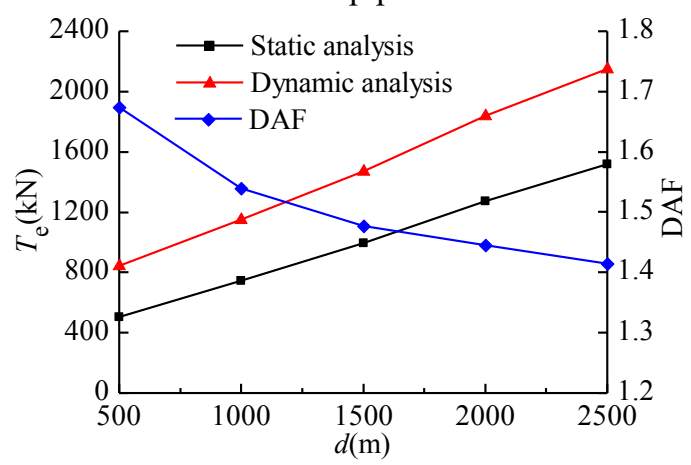

(b) Maximum axial tension of the pipeline

shown in Figure 10, as the stinger length increases, the axial tension of the entire pipeline has a slight increase, the maximum axial tension of the pipeline for static and dynamic analysis and its DAF mildly increase. In summary, the stinger length has little influence on the axial tension, and the increase of stinger length slightly enhances the dynamic lay effect on maximum axial tension of the pipeline.

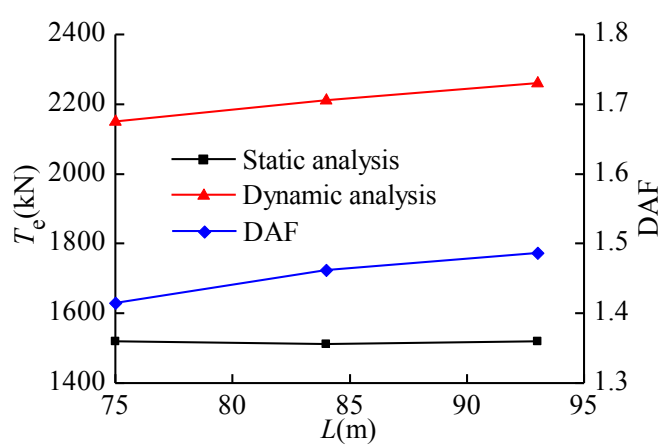

(b) Maximum axial tension of the pipeline

(a) Axial tension distribution along the pipeline

Figure 10 Axial tension response of the pipeline varied with stinger length 


\subsection{Tension Varied with Pipe Diameter}

In order to explore the axial tension response of the pipeline varied with pipe diameter, a group of steel pipes with outer diameters $8.625,10.75,12.75,14.00$ and 16.00 inch. are selected in terms of the API standard [12]. The corresponding wall thicknesses are 22.2, 20.6, 20.6, 20.6 and $22.2 \mathrm{~mm}$, and the submerged self-weights of the pipe per unit length are nearly the same and equal to 0.676 , $0.667,0.680,0.668$ and $0.756 \mathrm{kN} / \mathrm{m}$, respectively. The

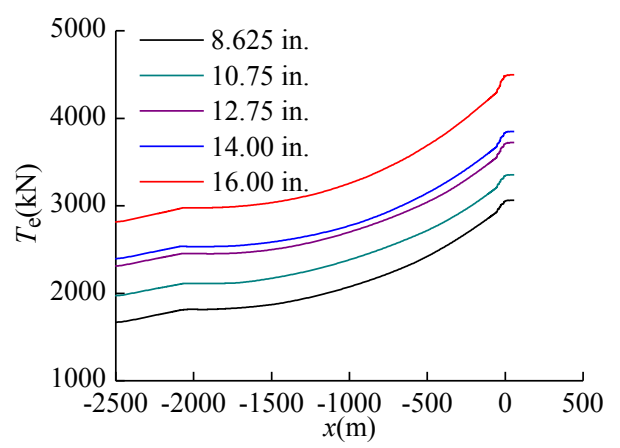

(a) Axial tension distribution along the pipeline axial tension responses of offshore pipeline laid into a water depth of $1500 \mathrm{~m}$ are shown in Figure 11. As the pipe diameter increases, the axial tension of the pipeline becomes apparently larger, the maximum axial tension of the pipeline for static analysis changes very small, while considering the dynamic lay effect, the maximum axial tension of the pipeline for dynamic analysis and its DAF remarkably raise. These results adequately demonstrated that the pipe diameter strongly influences the axial tension of the pipeline, and the increase of pipe diameter obviously enhances the dynamic lay effect on the maximum axial tension.

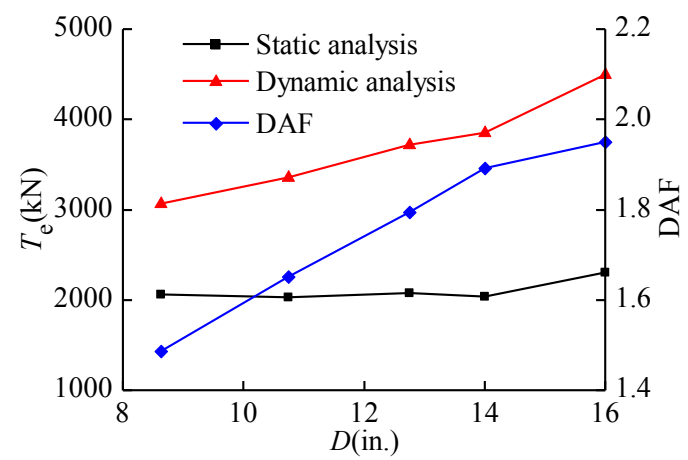

(b) Maximum axial tension of the pipeline

Figure 11 Axial tension response of the pipeline varied with pipe diameter

\subsection{Tension Varied with Submerged Self-weight of the Pipe}

To investigate the tension response of offshore pipeline varied with submerged self-weight of the pipe, a group of steel pipes with outer diameter $323.9 \mathrm{~mm}$, and wall thickness 19.1, 20.6, 23.8, 27.0 and $31.8 \mathrm{~mm}$ are selected in accordance with the standard API [12]. The corresponding submerged self-weights of the pipe per unit length are $0.58,0.68,0.90,1.11$ and $1.41 \mathrm{kN} / \mathrm{m}$, respectively. As shown in Figure 12, the axial tension of the pipeline significantly increases with the increase of submerged self-weight of the pipe, the maximum axial tension of the pipeline for both static and dynamic analysis linearly increases, whereas the DAF rapidly drops. In summary, the submerged self-weight of the pipe has a strong influence on axial tension of the pipeline, and the increase of submerged self-weight of the pipe apparently weakens the dynamic lay effect on the maximum axial tension.

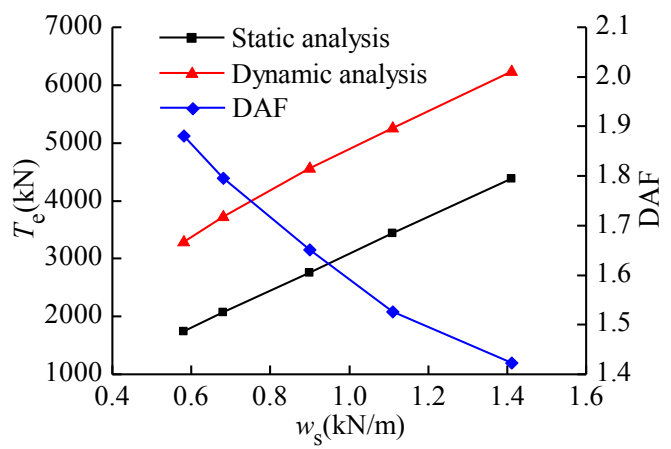

(b) Maximum axial tension of the pipeline

(a) Axial tension distribution along the pipeline Figure 12 Axial tension response of the pipeline varied with submerged self-weight of the pipe

\section{Conclusion}

This paper presents a full finite element model for deepwater S-lay system to explore the dynamic tension behaviour of offshore pipeline varied with various pipelay parameters. A strong relevance between pipelay parameters and axial tension of the pipeline is observed. These results show that the axial tension responses of the pipeline are greatly influenced by water depth, pipe diameter, and submerged self-weight of the pipe. Therefore, the design of the tensioners should sufficiently take into account the variations of the pipe tension under 
different pipelay parameters.

\section{Acknowledgements}

The authors express their gratitude to the National Natural Science Foundation of China (Grant nos. 51479176, 51679042), Youth Teacher Education Scientific Research Project in the Education Department of Fujian Province (Grant no. JAT170064), and Talent Introduction Scientific Start-up Foundation of Fuzhou University (Grant no. 510434) for the financial support to this study.

\section{References}

1. Li Z G, Wang C, He N, et al. An overview of deepwater pipeline laying technology[J]. China Ocean Engineering, 2008, 22(3): 521-532.

2. Clauss G F, Weede H, Riekert T. Offshore pipe laying operations-Interaction of vessel motions and pipeline dynamic stresses[J]. Applied Ocean Research, 1992, 14(3): 175-190.

3. Yun H D, Peek R R, Paslay P R, et al. Loading history effects for deepwater S-lay of pipelines[J]. Journal of Offshore Mechanics and Arctic Engineering, 2004, 126(2): 156-163.

4. O'Grady R, Harte A. Localised assessment of pipeline integrity during ultra-deep S-lay installation[J]. Ocean Engineering, 2013, 68: 27-37.

5. Mattiazzo G, Mauro S, Guinzio P S. A tensioner simulator for use in a pipelaying design tool[J]. Mechatronics, 2009, 19(8):1280-1285.

6. Yue Q J, Lu Q Z, Yan J, et al. Tension behavior prediction of flexible pipelines in shallow water[J]. Ocean Engineering, 2013, 58(2):201-207.

7. Gong S F, Xu P, Bao S, et al. Numerical modelling on dynamic behaviour of deepwater S-lay pipeline[J]. Ocean Engineering, 2014, 88: 393-408.

8. Gong S F, Xu P. The influence of sea state on dynamic behaviour of offshore pipelines for deepwater Slay[J]. Ocean Engineering, 2016, 111: 398-413.

9. Orcina. OrcaFlex User Manual, Version 9.7a[M]. Cumbria, UK: 2014.

10. Ramberg W, Osgood W R. Description of stress-strain curves by three parameters[C]. National Advisory Committee for Aeronautics (NACA) Technical Note, No.902, NACA, Washington, 1943.

11. Randolph M F, Quiggin P. Non-linear hysteretic seabed model for catenary pipeline contact[C]. In: Proceedings of the International Conference on Offshore Mechanics and Arctic Engineering, OMAE2009-79259, Hawaii, USA, 2009.

12. American Petroleum Institute (API). API Specification 5L: Specification for Line Pipe [S]. Washington: API Publishing Services, 2013. 